\title{
Evaluación de tres híbridos de maíz con niveles nitrogenados durante la estación lluviosa, Guayaquil, Ecuador
}

\author{
Evaluation of three corn hybrids with nitrogen levels during rainy season, \\ Guayaquil, Ecuador
}
Avaliação de três híbridos de milho com níveis nitrogenados durante a estação chuvosa, Guayaquil, Equador

- Jaime Proaño ${ }^{1}$

jproanos@espol.edu.ec

ORCID: 0000-0003-2980-5796
- Kentaro Tomita ${ }^{1,2}$

ktomita@espol.edu.ec

ORCID: 0000-0001-9000-4758

1Facultad de Ciencias de la Vida, Escuela Superior Politécnica del Litoral (ESPOL). Guayaquil, Ecuador. 2 Voluntario Senior de Agencia de Cooperación Internacional del Japón: JICA.

\section{RESUMEN}

Se evaluaron tres híbridos de maíz bajo tres niveles de fertilización nitrogenada $(50,100 \mathrm{y}$ $150 \mathrm{~kg}$ de $\mathrm{N} / \mathrm{ha}$ ) durante la estación lluviosa 2019 en la Granja Experimental Agrícola de la ESPOL (Escuela Superior Politécnica del Litoral), Guayaquil, Guayas, Ecuador. El suelo donde se implementó el experimento se clasifica como Vertisol y es conocido como de alta fertilidad, teniendo en cuenta su alto contenido de mineral arcilloso tipo 2:1 tales como la montmorillonita y/o ilita. Con una CIC y saturación de bases en la superficie son de 43,9meq/100g y 99,4\%, respectivamente. Los híbridos que se experimentaron son: Pioneer 4039 (P4039), Advanta 9313 (AV9313) y Advanta 9139 (AV9139), distribuidos por la compañía FARMAGRO. En el área experimental, se aplicaron tres niveles de fertilización nitrogenada $\quad[50,100 \quad y$ $150 \mathrm{kgN} / \mathrm{ha}$ ), $50 \mathrm{~kg}$ de P2O5 y $50 \mathrm{~kg}$ de K20/ha, respectivamente. Como característica agronómica, se observó diferencia significativa al $1 \%$ no sólo para el híbrido sino también para el nitrógeno, así como en la longitud de hoja, obteniendo el más alto valor para el AV 9139. Al igual que el caso anterior, el rendimiento del grano con 14\% de humedad, se observó una diferencia significativa al 5\% para la interacción. Aunque el más alto rendimiento fue para el AV9139, se observó una tendencia negativa de acuerdo a la mayor dosis de nitrógeno. Recomendándose el híbrido AV 9139 con la aplicación de $50 \mathrm{kgN} / \mathrm{ha}$ de acuerdo con los resultados obtenidos en el análisis económico.

Palabras clave: Aplicación económica; Beneficio neto; Fertilización nitrogenada; Híbrido; Vertisol
ABSTRACT

It was evaluated three hybrids of corn under the three levels of the nitrogen application $(50,100$ and $500 \mathrm{kgN} / \mathrm{ha})$ during the rainy season at the Experimental Field in the ESPOL (Escuela Superior Politécnica del Litoral) Guayaquil, Guayas, Ecuador. The soil where the experiment was implemented is classified as Vertisol and it is known as high fertility, taking into account its high content of type 2 : 1 clay mineral such as montmorillonite and or illite. It was $43,9 \mathrm{meq} / 100 \mathrm{~g}$ for CEC and $99,4 \%$ for the base saturation, respectively. These hybrids which were experimented are: Pioneer 4039 (P4039), Advanta 9313 (AV9313) and Advanta 9139 (AV9139) distributed by the company FARMAGRO. On the experimental area, it was applied $50 \mathrm{kgP} 205$ and $50 \mathrm{kgK} 20 /$ ha, respectively. As agronomic character, it was observed the significant difference al $1 \%$ not only for the hybrid but also for nitrogen, as well as for length of leave, obtaining the largest for the AV 9139. Like the previous case of the grain yield with $14 \%$ moisture, it was observed the significant difference at $5 \%$ for the interaction. Although it was the highest performance for the AV9139, it was observed the negative trend in accordance with the higher nitrogen amounts. Recommending the hybrid AV 9139 with the application of $50 \mathrm{kgN} / \mathrm{ha}$ according to the results obtained with the highest net benefit.

Key words: Economic application; Hybrid Net profit; Nitrogen fertilization; Vertisol

\section{RESUMO}

Avaliaram-se três híbridos de milho sob três níveis de fertilização com nitrogênio $(50,100$ e $150 \mathrm{~kg}$ de N/ha) durante a estação chuvosa 2019 na Fazenda Experimental Agrícola da ESPOL (Escola Superior Politécnica do Litoral), Guayaquil, Guayas, Equador. O solo onde implantou-se o experimento é classificado como Vertissolo e é conhecido como de alta fertilidade, levando em conta seu alto teor de mineral argiloso tipo 2: 1 tais como a montmorilonita e/ou ilita. Com uma CTC e saturação por bases na superfície são de 43,9 meq/100g e 99,4\%, respectivamente. Os híbridos que foram experimentados são: Pioneer 4039 (P4039), Advanta 9313 (AV9313) e Advanta 9139 (AV9139), distribuídos pela empresa FARMAGRO. Na área experimental, aplicaram-se três níveis de adubação nitrogenada (50, 100 e $150 \mathrm{kgN} / \mathrm{ha}), 50 \mathrm{~kg}$ de $\mathrm{P} 205$ e 50kg de $\mathrm{K} 2 \mathrm{O} / \mathrm{ha}$, respectivamente. Como característica agronômica, observou-se foi diferença significativa ao $1 \%$ não só para o híbrido, mas também para o nitrogênio, assim como na longitude de folha, obtendo-se o maior valor para AV 9139. Ao igual que o caso anterior, o rendimento do grão com $14 \%$ de umidade, observou-se diferença significativa ao 5\% para a interação. Embora o mais alto rendimento foi para AV9139, observou-se uma tendência negativa de acordo com a maior dose de nitrogênio. Recomendando-se o híbrido $\mathrm{AV}$ 9139 com a adubação de $50 \mathrm{kgN} /$ ha de acordo com os resultados obtidos na análise econômica.

Palavras-chave: Adubação econômica; Adubação nitrogenada; Híbrido; Lucro líquido; Vertissolo 


\section{INTRODUCCIÓN}

La calidad del grano de maíz para el consumo humano está asociada tanto con su constitución física, que determina la textura y dureza, como a su composición química, que define el valor nutricional y las propiedades tecnológicas. Los mercados son cada vez más exigentes y se interesan por el contenido de proteínas, aminoácidos, almidón, aceites y demás componentes.

El Maíz es una planta de fácil desarrollo y de producción anual, pertenece al género de las Zeas, de nombre científico Zea mays, familia de las gramíneas. El maíz amarillo duro (tipo cristalino) que se produce en Ecuador, es de excelente calidad tanto para la elaboración de alimentos balanceados como para las industrias de consumo humano; debido a su elevado contenido de fibra, carbohidratos, caroteno y el alto nivel de rendimiento en la molienda, así como por sus precios, nuestro maíz es de gran aceptación en países fronterizos.

De la producción nacional de maíz, la avicultura consume el 57\%, alimentos balanceados para otros animales 6\%, exportación a Colombia 25\%, industrias de consumo humano $4 \%$, el resto sirve para el autoconsumo y semilla. Además, Ecuador tiene la capacidad de exportar subproductos del maíz, tales como la sémola. Estos productos son utilizados para elaborar polenta, arepas y snacks.

\section{MATERIALES Y MÉTODOS}

Se sembraron tres híbridos maíz bajo tres niveles de fertilización nitrogenada $(50,100$ y $150 \mathrm{~kg} \mathrm{~N} / \mathrm{ha}$ ) durante la estación lluviosa del año 2019 en la Granja experimental de la ESPOL (Escuela Superior Politécnica del Litoral), Guayaquil, Guayas, Ecuador.

El orden de suelo encontrado en el área experimental corresponde a un Vertisol y es conocido por su alta fertilidad, con un alto contenido de mineral arcilloso tipo 2:1 tales como la montmorillonita y/o ilita.

Como híbridos, se utilizaron el Pioneer 4039 (P4039), Advanta 9313 (AV9313) y Advanta 9139 (AV9139), respectivamente.

El área experimental es de $734,4 \mathrm{~m}^{2}$ y se separaron 9 subparcelas de $81,6 \mathrm{~m}^{2}$, y se sortearon los tres híbridos con tres repeticiones. Además, en cada subparcela se separaron tres subparcelas de $27,2 \mathrm{~m}^{2}$, con los tres niveles de fertilización nitrogenada (50, 100 y $150 \mathrm{kgN} / \mathrm{ha}$ ) al azar (31 y 81 y $139 \mathrm{kgN} / \mathrm{ha}$, usando Bonaza nitro cuyo contenido de $38 \% \mathrm{~N}$ ). Para la fertilización nitrogenada, se separó tres épocas como 7, 30 y 60 días después de la siembra. (2).

Para la fertilización fosfatada, se utilizó el fertilizante comercial DAP $\left(18 \% \mathrm{~N}-46 \% \mathrm{P}_{2} \mathrm{O}_{5}-\right.$ $0 \% \mathrm{~K}_{2} \mathrm{O}$ ) aplicándose $50 \mathrm{kgP}_{2} \mathrm{O}_{5} / \mathrm{ha}$ en cada tratamiento. Al mismo tiempo, se aplicó $19 \mathrm{kgN} / \mathrm{ha}$ en todos los tratamientos por el DAP, totalmente, fue de 50, 100 y $150 \mathrm{kgN} / \mathrm{ha}$ en cada tratamiento nitrogenado. (3).

Para la fertilización potásica se aplicaron $50 \mathrm{kgK}_{2} \mathrm{O} / \mathrm{ha}$, usando el $\mathrm{KCl}\left(0-0-60 \% \mathrm{~K}_{2} \mathrm{O}\right)$, al igual que el caso del Bonanza nitro, se lo aplicó, separando las tres épocas.

La siembra del maíz se realizó a una distancia de $80 \mathrm{~cm}$ entre hileras y $20 \mathrm{~cm}$ entre plantas, dejando una planta por hoyo, para una población de 62500 plantas/ha.

Para el control de arvenses se aplicó $150 \mathrm{ml}$ del herbicida paraquat por 20L de agua.

Antes de la cosecha, se realizó la evaluación de las características agronómicas, tales como: altura de planta, inserción de mazorca y longitud de hoja, al azar con 10 plantas cada dos hileras.

Se realizó la cosecha en el área útil $(2,0 \mathrm{~m} x$ $1,6 \mathrm{~m}=3,2 \mathrm{~m}^{2}$ ). Una vez obtenida una humedad del grano del $14 \%$, se pesaron los tratamientos 
convirtiéndolos a $\mathrm{kg} / \mathrm{ha}$, usando los rendimientos obtenidos en el área útil.

Luego, se realizó el tratamiento estadístico (Arreglo en parcelas divididas sobre Bi factoriales), para altura de planta $(\mathrm{cm})$, inserción de la mazorca $(\mathrm{cm})$ y longitud de hoja (cm) como característica agronómica y el rendimiento del grano (kg/ha), respectivamente. experimental en la ESPOL. Al inicio del mes de noviembre del 2018 se describió la calicata del suelo para que conocer las características fisicoquímicas del suelo cultivado.

Para confirmar la diversidad del suelo en el área de la Granja experimental, se analizó el sitio donde cultivaron los tres híbridos. La Tabla No1 muestra la dinámica de cada nutriente en el suelo $(0-50 \mathrm{~cm}$ de la profundidad).

\section{RESULTADOS}

\section{Propiedad química del suelo antes de la siembra y fertilización}

Se clasifica el suelo como un Vertisol (Taxonomía del suelo por USA) en la Granja

Tabla 1. Dinámica de cada nutriente en el suelo $(0-50 \mathrm{~cm}$ de la profundidad) antes de la aplicación y siembra.

\begin{tabular}{|c|c|c|c|c|c|c|c|c|c|c|}
\hline \multirow{3}{*}{$\begin{array}{l}\text { Muestra del suelo } \\
\text { Profundidad }\end{array}$} & \multirow{3}{*}{$\begin{array}{c}\mathrm{pH} \\
\\
\mathrm{H}_{2} \mathrm{O} \\
\mathbf{1} ; \mathbf{1}\end{array}$} & \multirow{3}{*}{$\begin{array}{c}\text { EC } \\
1 ; 1 \\
\text { (uS/cm) }\end{array}$} & \multirow{3}{*}{$\begin{array}{c}\text { Disponible } \\
\text { P } \\
\text { (mg/L) }\end{array}$} & \multicolumn{4}{|c|}{ Intercambiable } & \multirow[t]{3}{*}{ CIC } & \multirow{3}{*}{$\begin{array}{c}\begin{array}{c}\text { Sat. } \\
\text { Bases }\end{array} \\
(\%)\end{array}$} & \multirow{3}{*}{$\begin{array}{l}\text { MO } \\
\text { (\%) }\end{array}$} \\
\hline & & & & $\mathbf{K}$ & $\mathrm{Ca}$ & Mg & $\mathrm{Na}$ & & & \\
\hline & & & & \multicolumn{4}{|c|}{$\mathrm{cmol}_{\mathrm{c}} / \mathrm{kg}$} & & & \\
\hline $0-15 \mathrm{~cm}$ & 6.88 & 82.3 & 0.501 & 0.716 & 24.7 & 14.1 & 0.182 & 39.9 & 99.3 & 5.36 \\
\hline $15-30 \mathrm{~cm}$ & 6.89 & 60.7 & 0.621 & 0.687 & 23.8 & 14.0 & 0.208 & 38.9 & 99.5 & 5.16 \\
\hline $30-50 \mathrm{~cm}$ & 7.33 & 25.6 & 0.218 & 0.289 & 29.7 & 18.9 & 0.576 & 49.7 & 99.5 & 3.94 \\
\hline
\end{tabular}

\section{pH $\left(\mathrm{H}_{2} \mathrm{O}\right)$}

Se observó cerca de 7 para el pH en la superficie, pero, para $50 \mathrm{~cm}$ de la profundidad, más de 7 en el suelo. Básicamente, se considera la acumulación de bases, teniendo en cuenta la característica mineral arcilloso con muy alta fertilidad en el suelo.

\section{CE (Conductividad Eléctrica)}

Relativamente, se observó alto valor en la superficie, de acuerdo con la profundidad, se disminuyó para la $\mathrm{CE}$, relativamente.

\section{Materia orgánica}

Relativamente, se observó alto valor en la superficie, de acuerdo con la profundidad, se disminuyó para la MO, relativamente. En realidad, se observó alto valor para la MO en los suelos minerales, se consideró muy alta fertilidad en el suelo Vertisol.

\section{$P$ disponible}

Se observó el valor más alto a $30 \mathrm{~cm}$ de profundidad con respecto al valor en la superficie. Se considera una alta influencia de 
la materia orgánica como biomasa residual y formar quelato con el $\mathrm{P}$ aplicado, lixiviándose a mayor profundidad.

\section{CIC (Capacidad de Intercambio} Catiónico)

Obteniéndose valores altos para la CIC, por encontrarse con arcilla del tipo 2:1 tales como la montmorillonita e ilita principalmente.

\section{Ca intercambiable}

Se observaron valores altos en todas las profundidades.

\section{Mg intercambiable}

$\mathrm{Al}$ igual que caso anterior se observaron valores altos en todas las profundidades. Se considera una alta relación con el valor de la CIC (ver la Figura No5).

\section{K intercambiable}

A diferencia del caso cálcico y magnésico, se observó muy bajo valor en todas las profundidades.

\section{Na intercambiable}

También, se realizó el análisis del $\mathrm{Na}$ intercambiable. Fue más bajo para el Na que el valor potásico en el suelo. Pero, a diferencia del caso potásico, aumentó para el valor de acuerdo con la profundidad. No podría ignorar la salinidad del suelo.

\section{Característica agronómica para el maíz}

\section{Altura de la planta}

La Figura 1 muestra la altura de cada híbrido para cada tratamiento nitrogenado. De los resultados del análisis de varianza, no se observó diferencia significativa.

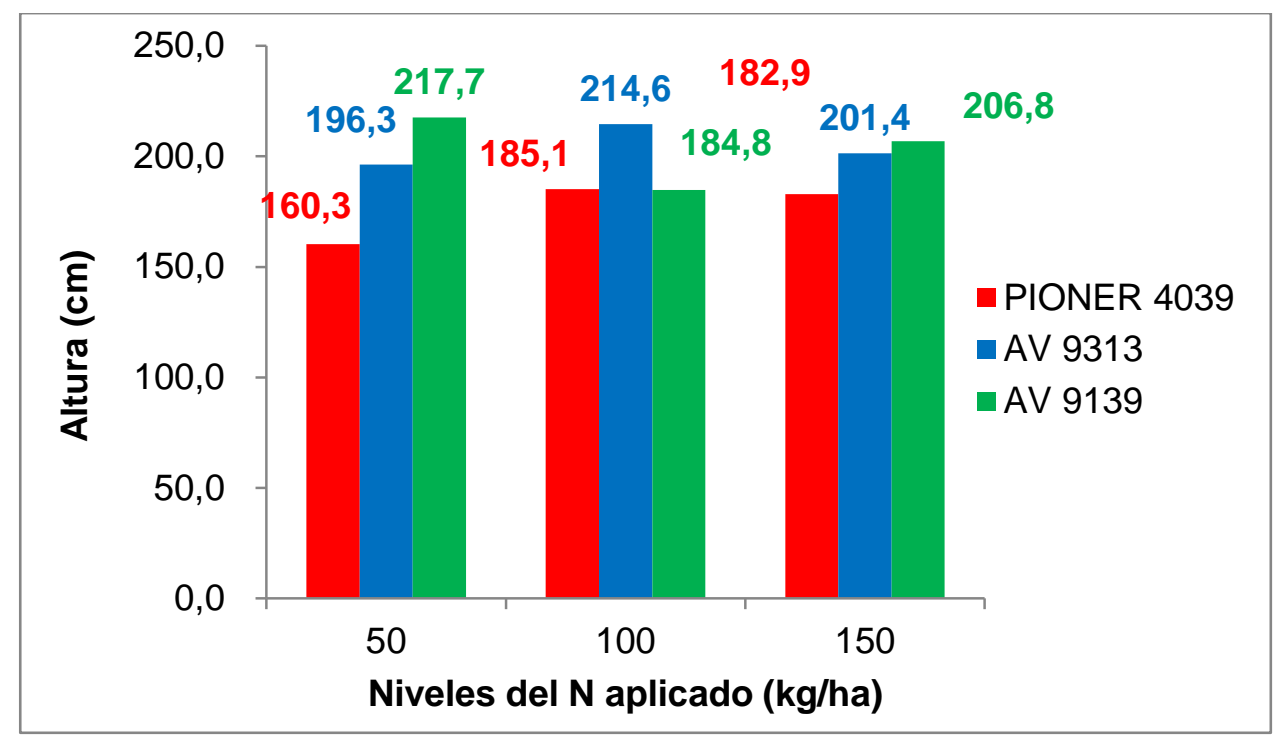

Figura 1. Comparación de la altura en cada híbrido y tratamiento nitrogenado. 
En realidad, se observó el más alto valor para el AV 9139 con $50 \mathrm{kgN} / \mathrm{ha}$, al aplicar 100 y $200 \mathrm{kgN} / \mathrm{ha}$, se redujo. El valor más bajo con respecto a la altura fue para el PIONER 4039.

\section{Longitud de la hoja}

La Figura 2 muestra la comparación de la longitud de hoja en cada híbrido y tratamiento nitrogenado. De los resultados del análisis de varianza, se observó la diferencia significativa al $1 \%$ para el híbrido e interacción.

Para la longitud de hoja, en realidad, se observó la más larga para el híbrido AV 9139 sin diferentes niveles del $\mathrm{N}$ aplicado.

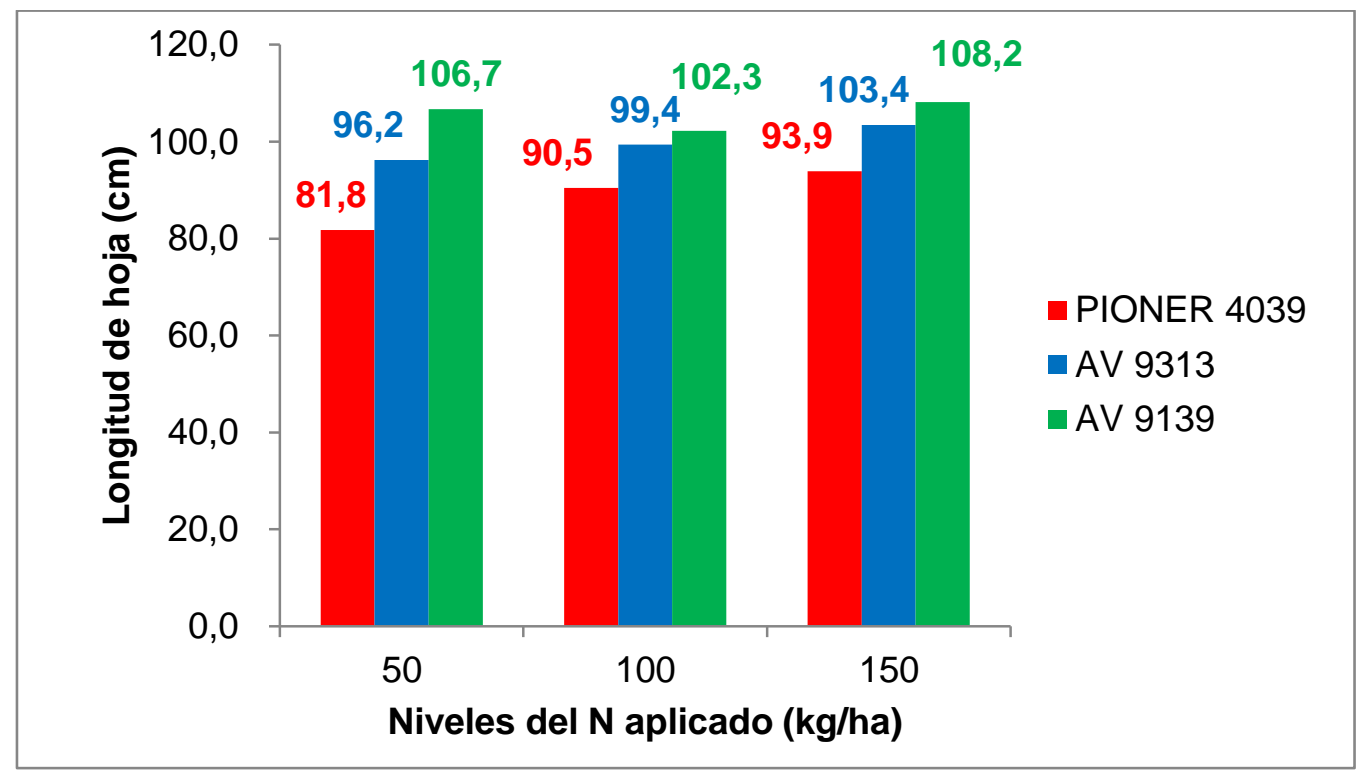

Figura 2. Comparación de la longitud de hoja en cada híbrido y tratamiento nitrogenado.

\section{Rendimiento del grano para el maíz}

\section{Área útil}

La Figura 3 muestra área útil para la evaluación del rendimiento del grano. Seleccionando dos hileras de $1,6 \mathrm{~m} \times 2,0 \mathrm{~m}=3,2 \mathrm{~m}^{2}$ como el área útil. 


\begin{tabular}{|c|c|c|c|c|c|c|c|c|}
\hline & $0,4 \mathrm{~m}$ & $0,4 \mathrm{~m}$ & $0,8 \mathrm{~m}$ & $0^{0,4 m}$ & $0,4 \mathrm{~m}$ & $0,8 \mathrm{~m}$ & $0,4 \mathrm{~m}$ & $0,4 \mathrm{~m}$ \\
\hline $0,2 \mathrm{~m}$ & 0 & 0 & & 0 & 0 & & 0 & 0 \\
\hline $0,2 \mathrm{~m}$ & 0 & 0 & & 0 & 0 & & 0 & 0 \\
\hline $0,2 \mathrm{~m}$ & 0 & ○ & & 0 & 0 & & 0 & 0 \\
\hline $0,2 \mathrm{~m}$ & 0 & 0 & & 0 & 0 & & 0 & 0 \\
\hline $0,2 \mathrm{~m}$ & 0 & 0 & & 0 & 0 & & 0 & 0 \\
\hline $0,2 \mathrm{~m}$ & 0 & o & & 0 & 0 & & 0 & 0 \\
\hline $0,2 \mathrm{~m}$ & 0 & ○ & & 0 & o & & o & 0 \\
\hline $0,2 \mathrm{~m}$ & 0 & o & & 0 & o & & 0 & 0 \\
\hline $0,2 \mathrm{~m}$ & 0 & o & & o & ○ & & 0 & 0 \\
\hline $0,2 \mathrm{~m}$ & 0 & o & & 0 & ○ & & 0 & 0 \\
\hline , 211 & 0 & 0 & & 0 & 0 & & 0 & ○ \\
\hline $0,2 \mathrm{~m}$ & o & o & & o & o & & 0 & 0 \\
\hline $0,2 \mathrm{~m}$ & 0 & 0 & & 0 & o & & 0 & 0 \\
\hline $0,2 \mathrm{~m}$ & 0 & 0 & & 0 & 0 & & 0 & 0 \\
\hline
\end{tabular}

Figura 3. Área útil en cada tratamiento.

\section{Resultados estadísticos}

La Figura 4 muestra la dinámica del rendimiento del grano con $14 \%$ de humedad en cada híbrido, con diferentes niveles del $\mathrm{N}$ aplicado.

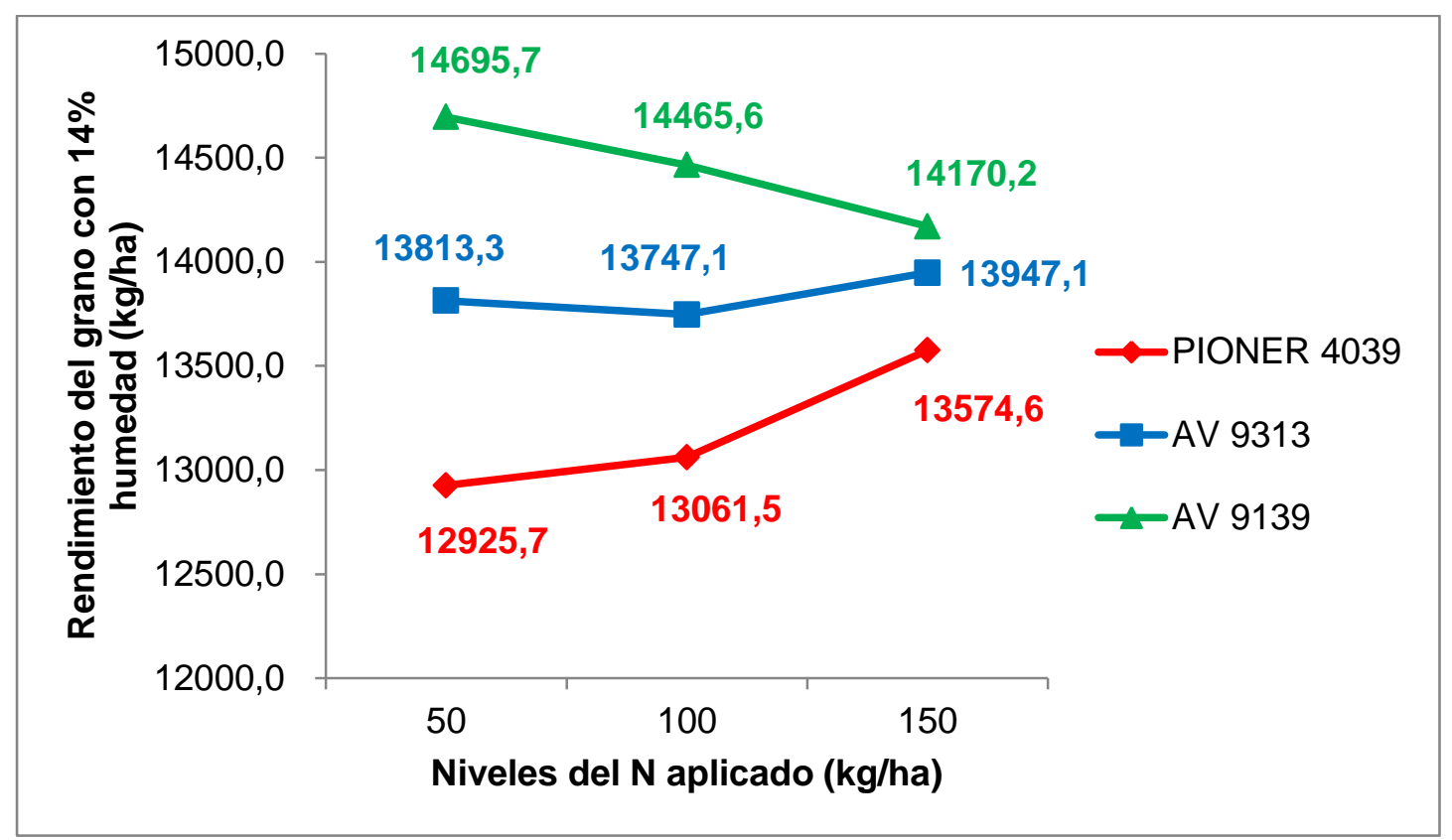

Figura 4. Dinámica del rendimiento del grano con $14 \%$ de humedad en cada híbrido, con diferentes niveles del $\mathrm{N}$ aplicado. 
De los resultados del análisis de varianza, se observó la diferencia significativa al 5\% para la interacción. En realidad, para el híbrido PIONEER 4029, aumentó el rendimiento, mientras que para el AV 9139 se redujo de acuerdo con la aplicación nitrogenada. Por otra parte, para el AV 9313, no reaccionó con diferentes niveles del $\mathrm{N}$ aplicado y fue de alrededor de $13700 \mathrm{~kg} / \mathrm{ha}$ como promedio del rendimiento. Se observó el más alto rendimiento para el AV 9139 con la aplicación del $50 \mathrm{kgN} / \mathrm{ha}$.

\section{Evaluación económica para el cultivo de maíz}

Es muy importante que se evalúe no sólo la producción agrícola sino también la estimación económica para el cultivo de maíz en un suelo Vertisol con el fin de establecer un sistema de la producción sustentable. (1).

Se explica la evaluación económica en cada tratamiento nitrogenado en cada híbrido. A continuación, sigue la evaluación económica para el cultivo de maíz.

Tabla 2. Costo fijo para el cultivo de maíz.

\begin{tabular}{|c|c|c|c|c|c|}
\hline \multicolumn{6}{|c|}{ PRESUPUESTO DEL CULTIVO DE MAIZ } \\
\hline \multicolumn{6}{|l|}{ Costo fijo } \\
\hline \multicolumn{6}{|l|}{ Preparación del terreno } \\
\hline \multicolumn{2}{|l|}{ Actividades } & Cantidad & \multicolumn{2}{|c|}{$\begin{array}{l}\text { Precio Unitario } \\
\text { (ha) }\end{array}$} & Costo (US\$) \\
\hline \multicolumn{2}{|l|}{ Arada } & 2 & \multicolumn{2}{|c|}{25} & 50 \\
\hline \multirow[t]{2}{*}{ Rastreada } & & 2 & \multicolumn{2}{|c|}{25} & 50 \\
\hline & & & \multicolumn{2}{|c|}{ Sub Total } & 100 \\
\hline \multicolumn{6}{|l|}{ Mano de obra } \\
\hline Actividades a realizar & Cantidad & $\begin{array}{l}\text { Días de } \\
\text { trabajo }\end{array}$ & $\begin{array}{c}\text { No. de } \\
\text { personales }\end{array}$ & $\begin{array}{l}\text { Precio } \\
\text { jornal }\end{array}$ & Costo (US\$) \\
\hline Siembra & 8 & 1 & 8 & 20 & 160 \\
\hline Aplicación Herbicidas & 2 & 1 & 2 & 20 & 40 \\
\hline Aplicación Insecticidas & 4 & 1 & 4 & 20 & 80 \\
\hline Aplicación Fertilizantes & 8 & 1 & 8 & 20 & 160 \\
\hline \multirow[t]{2}{*}{ Cosecha } & 8 & 2 & 4 & 20 & 160 \\
\hline & & & & Sub Total & 600 \\
\hline
\end{tabular}




\begin{tabular}{lcccc}
\hline Insumo & \multicolumn{3}{c}{} & \\
\hline Productos & Cantidad & Precio (US\$) & Presentación & Costo (US\$) \\
PICADOR & 1 & 14.0 & $100 \mathrm{cc}$ & 14.0 \\
SADDLER & 1 & 9.2 & $250 \mathrm{cc}$ & 9.2 \\
ZEAMAX & 3 & 13.2 & $32 \mathrm{~g}$ & 39.5 \\
GLIFOSATO & 1 & 5.1 & $1 \mathrm{Lt}$ & 5.1 \\
LORSBAN LOW & 1 & 13.8 & $1 \mathrm{Lt}$ & 13.8 \\
PREMIO & 1 & 39.3 & $100 \mathrm{cc}$ & 39.3 \\
MITERRA & 1 & 16.2 & $250 \mathrm{cc}$ & 16.2 \\
RADIFLEX & 1 & 17.9 & $1 \mathrm{Lt}$ & 17.9 \\
COSMOAGUAS & 1 & 10.0 & $1 \mathrm{~kg}$ & 10.0 \\
COSMO IND & 1 & 12.4 & $1 \mathrm{Lt}$ & 12.4 \\
GLORY & 1 & 14.5 & $1 \mathrm{Kg}$ & 14.5 \\
YOKE & 1 & 15.1 & $500 \mathrm{cc}$ & 15.1 \\
PLEO & 1 & 26.2 & $75 \mathrm{cc}$ & 26.2 \\
TEJO & 1 & 23.8 & $150 \mathrm{~g}$ & 23.8 \\
FIDELUTY & 1 & 14.4 & $200 \mathrm{cc}$ & 14.4 \\
BIOTEK & 1 & 11.2 & $200 \mathrm{cc}$ & 11.2 \\
FARMAVERDOL & 1 & 12.9 & $1 \mathrm{Lt}$ & 12.9 \\
COSMO MADURARDOR & 1 & 31.5 & $1 \mathrm{Kg}$ & 31.5 \\
DAD(18-46-0) : 50gg/bolsa & 2,2 bolsas & 1.4 & $50 \mathrm{~kg} \mathrm{como} \mathrm{P}_{2}$ & 68.4 \\
KCI (0-0-60) : 50kg/bolsa & 1,7 bolsas & 0.8 & $50 \mathrm{~kg} \mathrm{como} \mathrm{K}_{2} \mathrm{O}$ & 38.3 \\
& & & Sub Total & $\mathbf{4 3 4}$ \\
& & & Costo fijo & $\mathbf{1 3 4}$ \\
\hline
\end{tabular}

\section{Costo fijo}

La Tabla 2 muestra el costo fijo para el cultivo de maíz que no se incluye el costo de cada híbrido ni el fertilizante nitrogenado. Para precio de los híbridos, se incluye al costo variable porque fue diferente para el precio sobre el híbrido utilizado con el fertilizante.

Es conocido la alta fertilidad para el suelo Vertisol, teniendo en cuenta alta donación de mineral arcilloso tipo 2:1 tal como montmorillonita e ilita. Fue necesario realizar varias aplicaciones de productos químicos, por lo que fue de US\$1134 para el costo fijo.

\section{Costo del híbrido}

La Tabla 3 muestra el costo variable para cada híbrido por hectárea. Como unidad, se calculó para 60000 semillas en cada híbrido. Por buena suerte, se realizó el descuento (15\%), se lo calculó como costo variable sobre híbrido. 
Tabla 3. Costo variable para el precio de cada híbrido

\begin{tabular}{lccc}
\hline Insumo & & & \\
\hline Híbrido & Cantidad (kg/ha) & Precio (US\$/Kg) & Descuento (15\%) \\
P 4039 & 60000 semillas & 250,00 & 212,5 \\
AV 9313 & 60000 semillas & 258,00 & 219,3 \\
AV 9139 & 60000 semillas & 278,00 & 236,3 \\
\hline
\end{tabular}

\section{Costo fijo total}

Como costo fijo total (costo fijo +60000 semillas),

P 4039: 1134 como costo fijo $+212,5$ como costo del P $4039=\mathbf{U S \$ 1 3 4 6 .}$

AV 9313: 1134 como costo fijo $+219,3$ como costo del AV9313 = US\$ 1353.

AV 9139: 1134 como costo fijo + 236,3 como costo del AV $9139=\mathbf{U S \$ 1 3 7 0 .}$

\section{Costo variable}

En realidad, para el Bonanza nitro, se aplicó 31, 81 y $131 \mathrm{kgN} / \mathrm{ha}$ en los tratamientos con 50, 100 y $150 \mathrm{kgN} / \mathrm{ha}$, teniendo en cuenta la aplicación del DAP como $50 \mathrm{kgP}_{2} \mathrm{O}_{5} /$ ha en todos los tratamientos. Como el costo variable de cada aplicación nitrogenada, fue de US\$ 47, 123 y 199/ha ${ }^{1}$ de acuerdo con 50,100 y $150 \mathrm{kgN} / \mathrm{ha}$, respectivamente.

\section{Precio de venta}

Según una información del Ministerio de Agricultura y Ganadería, el precio referencial en el año 2019 fue de US\$15,25 por quintal $(=100 \mathrm{Lbs}=45,36 \mathrm{~kg})$ con un $13 \% \mathrm{de}$ humedad. Por lo tanto, el costo por kilo fue de US\$0,34/kg.

\section{Evaluación de beneficio neto en cada tratamiento nitrogenado asociado con cada híbrido}

Para el experimento, se realizó de acuerdo con el análisis de varianza con bifactoriales tal como híbrido por aplicación nitrogenada.

La Tabla 4 muestra la evaluación económica para P 4039 (PIONEER 4039) en cada tratamiento nitrogenado. Básicamente, se pudo esperar alto beneficio neto, teniendo en cuenta alto rendimiento obtenidos en el suelo Vertisol.

\footnotetext{
${ }^{1}$ Bonanza nitro $(50 \mathrm{kgN} / \mathrm{ha})=$ US\$1,52kg por $31 \mathrm{kgN} / \mathrm{ha}=$ US\$47/ha, Bonanza nitro $(100 \mathrm{kgN} / \mathrm{ha})=$ US\$1,52/ha por $81 \mathrm{kgN} / \mathrm{ha}$ = US\$123/ha y Bonanza nitro $(150 \mathrm{kgN} / \mathrm{ha})=$ US\$1,52 por $131 \mathrm{kgN} / \mathrm{ha}=$ US\$199/ha, respectivamente.
}

Alfa, Revista de Investigación en Ciencias Agronómicas y Veterinarias Vol. 5, Nro. 13, Enero - abril 2021 
Tabla 4. Evaluación económica para P 4039 en cada tratamiento nitrogenado.

\begin{tabular}{ccccccccc}
\hline $\begin{array}{c}\mathbf{N} \\
\text { (kg/ha) }\end{array}$ & $\begin{array}{c}\text { Rendimiento } \\
\text { Estipulado } \\
\text { Kg/ha } \\
\text { (kg/ha) }\end{array}$ & $\begin{array}{c}\mathbf{2 0 \%} \\
\text { perdida en } \\
\text { la cosecha } \\
\text { (kg/ha) }\end{array}$ & $\begin{array}{c}\text { Kg a ser } \\
\text { vendido } \\
\text { (kg/ha) }\end{array}$ & $\begin{array}{c}\text { Beneficio } \\
\text { bruto } \\
\text { (US\$/ha) }\end{array}$ & $\begin{array}{c}\text { Costo } \\
\text { variable } \\
\text { (kg/ha) }\end{array}$ & $\begin{array}{c}\text { Costo fijo + } \\
\mathbf{6 0 0 0 0} \\
\text { semillas } \\
\text { (US\$/ha) }\end{array}$ & $\begin{array}{c}\text { Costo Total } \\
\text { (US\$/ha) }\end{array}$ & $\begin{array}{c}\text { Beneficio } \\
\text { neto } \\
\text { (US\$/ha) }\end{array}$ \\
\hline 50 & 12926 & 2585 & 10341 & 3102 & 47 & 1346 & 1393 & 1709 \\
100 & 13062 & 2612 & 10450 & 3135 & 123 & 1346 & 1469 & 1666 \\
150 & 13575 & 2715 & 10860 & 3258 & 199 & 1346 & 1545 & 1713 \\
\hline
\end{tabular}

Nota: Precio del grano de maíz fue de US $\$ 0.34 / \mathrm{kg}$, Costo del N $/ \mathrm{kg}$ fue de US $\$ 1.52$ (Costo de la Bonanza fue de US\$28,9/50kg). Además, Kg a ser vendido = rendimiento estipulado - $20 \%$ perdida en la cosecha.

Tabla 5. Evaluación económica para AV 9313 en cada tratamiento nitrogenado.

\begin{tabular}{ccccccccc}
\hline $\begin{array}{c}\mathbf{N} \\
\text { (kg/ha) }\end{array}$ & $\begin{array}{c}\text { Rendimiento } \\
\text { Estipulado } \\
\text { Kg/ha } \\
\text { (kg/ha) }\end{array}$ & $\begin{array}{c}\mathbf{2 0 \%} \\
\text { perdida en } \\
\text { la cosecha } \\
\text { (kg/ha) }\end{array}$ & $\begin{array}{c}\text { Kg a ser } \\
\text { vendido } \\
\text { (kg/ha) }\end{array}$ & $\begin{array}{c}\text { Beneficio } \\
\text { bruto } \\
\text { (US\$/ha) }\end{array}$ & $\begin{array}{c}\text { Costo } \\
\text { variable } \\
\text { (kg/ha) }\end{array}$ & $\begin{array}{c}\text { Costo fijo + } \\
\mathbf{6 0 0 0 0} \\
\text { semillas } \\
\text { (US\$/ha) }\end{array}$ & $\begin{array}{c}\text { Costo Total } \\
\text { (US\$/ha) }\end{array}$ & $\begin{array}{c}\text { Beneficio } \\
\text { neto } \\
\text { (US\$/ha) }\end{array}$ \\
\hline 50 & 13813 & 2763 & 11050 & 3315 & 47 & 1353 & 1400 & 1915 \\
100 & 13747 & 2749 & 10998 & 3299 & 123 & 1353 & 1477 & 1823 \\
150 & 13947 & 2789 & 11158 & 3347 & 199 & 1353 & 1553 & 1795 \\
\hline
\end{tabular}

Nota: Precio del grano de maíz fue de US $\$ 0.34 / \mathrm{kg}$, Costo del $\mathrm{N} / \mathrm{kg}$ fue de US $\$ 1.52$ (Costo de la Bonanza fue de US\$28,9/50kg). Además, Kg a ser vendido = rendimiento estipulado - $20 \%$ perdida en la cosecha.

Además de la evaluación para el híbrido P 4039, las Tablas №5 y No6 muestran la evaluación para AV 9313 y AV 9139, respectivamente.

Se obtuvo un beneficio neto más alto para los híbridos AV 9313 y 9139 con respecto al híbrido P 4039.

Tabla 6. Evaluación económica para AV 9139 en cada tratamiento nitrogenado.

\begin{tabular}{|c|c|c|c|c|c|c|c|c|}
\hline $\begin{array}{c}N \\
(\mathrm{~kg} / \mathrm{ha})\end{array}$ & $\begin{array}{c}\text { Rendimiento } \\
\text { Estipulado } \\
\mathrm{Kg} / \mathrm{ha} \\
\text { (kg/ha) }\end{array}$ & $\begin{array}{c}20 \% \\
\text { perdida en } \\
\text { la cosecha } \\
(\mathrm{kg} / \mathrm{ha})\end{array}$ & $\begin{array}{l}\text { Kg a ser } \\
\text { vendido } \\
\text { (kg/ha) }\end{array}$ & $\begin{array}{c}\text { Beneficio } \\
\text { bruto } \\
\text { (US } \$ / \text { ha) }\end{array}$ & $\begin{array}{c}\text { Costo } \\
\text { variable } \\
\text { (kg/ha) }\end{array}$ & $\begin{array}{c}\text { Costo fijo + } \\
60000 \\
\text { semillas } \\
\text { (US\$/ha) }\end{array}$ & $\begin{array}{c}\text { Costo Total } \\
\text { (US\$/ha) }\end{array}$ & $\begin{array}{c}\text { Beneficio } \\
\text { neto } \\
\text { (US\$/ha) }\end{array}$ \\
\hline 50 & 14696 & 2339 & 11757 & 3527 & 47 & 1370 & 4717 & 2110 \\
\hline 100 & 14466 & 2893 & 11573 & 3472 & 123 & 1370 & 1494 & 1978 \\
\hline 150 & 14170 & 2834 & 11336 & 3401 & 199 & 1370 & 1570 & 1831 \\
\hline
\end{tabular}

Nota: Precio del grano de maíz fue de US $\$ 0.34 / \mathrm{kg}$, Costo del N $/ \mathrm{kg}$ fue de US $\$ 1.52$ (Costo de la Bonanza fue de US\$28,9/50kg). Además, Kg a ser vendido = rendimiento estipulado - $20 \%$ perdida en la cosecha.

\section{Día de campo en el campo}

La Foto 1 muestra la condición del cultivo de maíz cerca de la cosecha. FARMAGRO. Ltd. Donó los tres. 


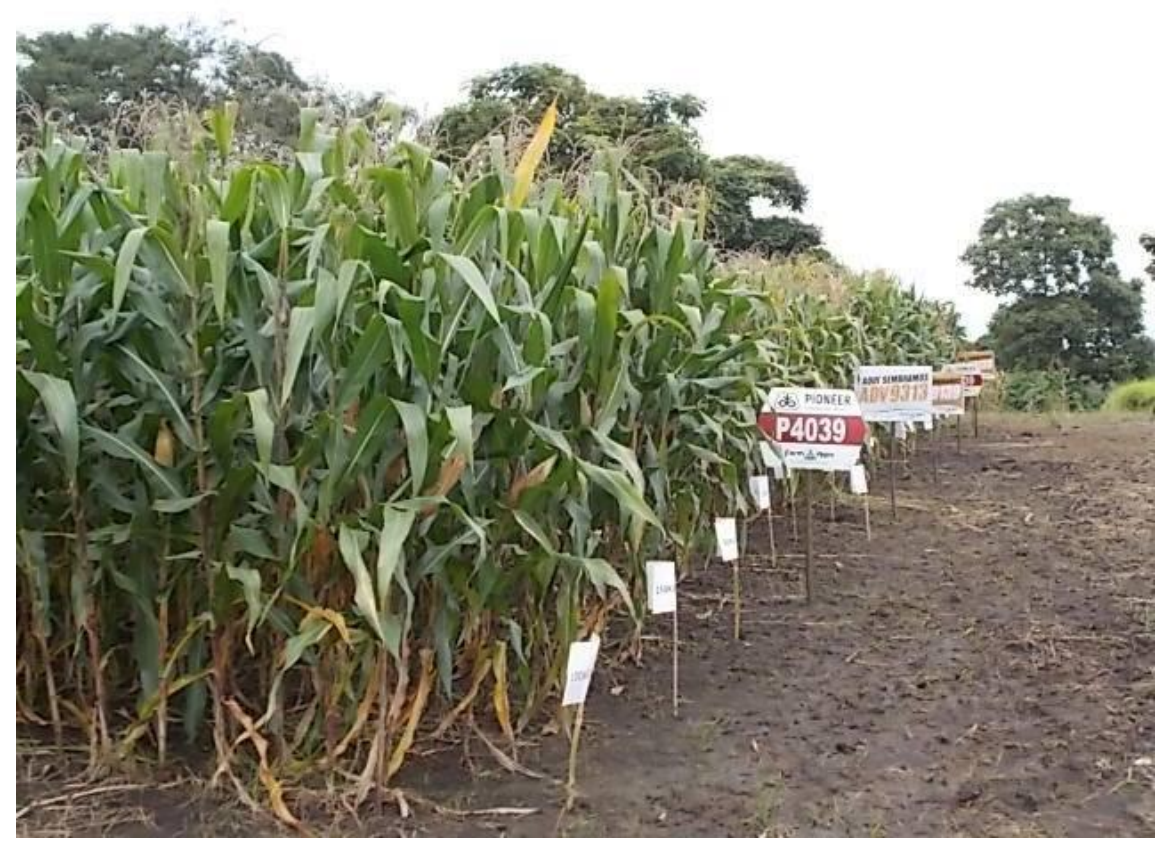

Foto 1. Condición del cultivo de maíz cerca de la cosecha en el día 22 de marzo del 2019.

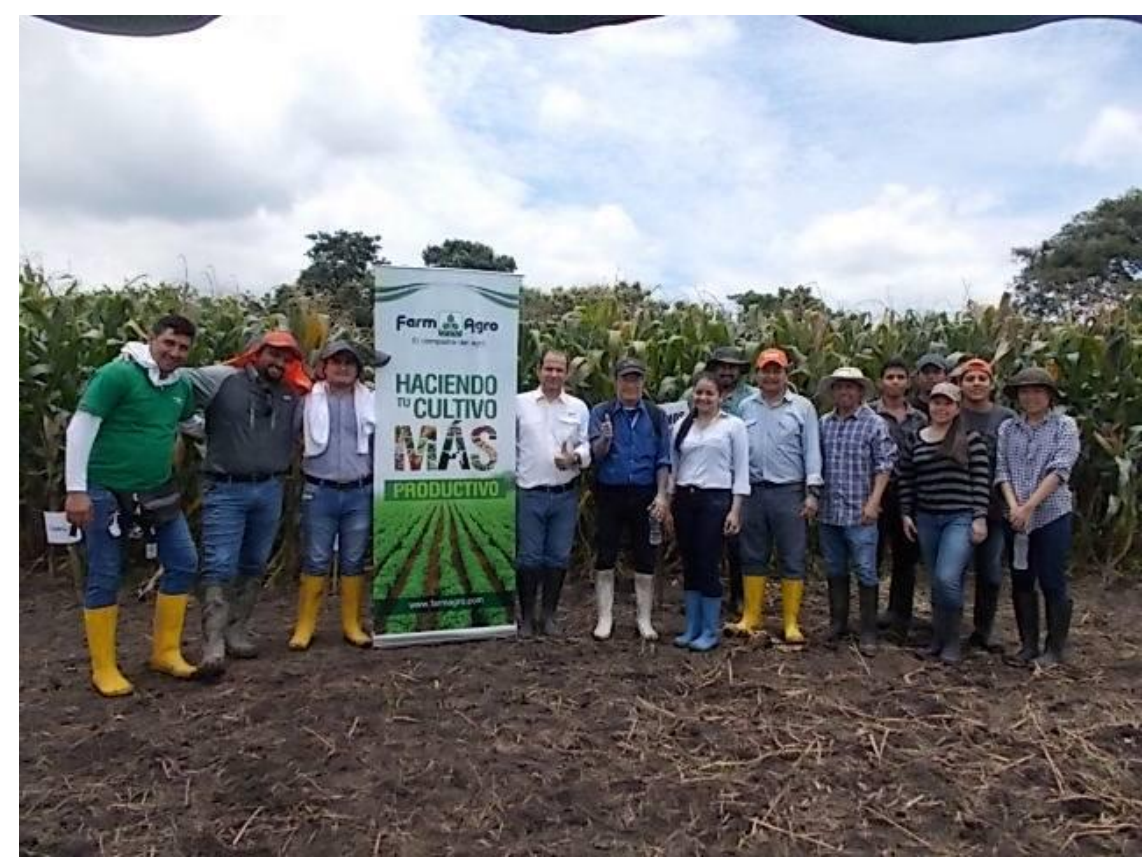

Foto 2. Participantes con los autores enfrente del cultivo de maíz en el día de campo.

Finalmente, la Foto 2 muestra a los participantes con los autores en el día de campo. Después de terminar la explicación en el día de campo. 
DISCUSIÓN

Es muy importante que establezca desde punta de vista no sólo mantenimiento $\mathrm{y} / \mathrm{o}$ aumento del rendimiento por manejo de la fertilidad del suelo, sino también prevención de alto coto para la producción agrícola sustentable, teniendo en cuenta escogiéndose híbrido adecuado con alta productividad.

Por eso, es necesario que realice el experimento en el campo para dibujar curva de respuesta para cada cultivo con diferentes niveles de la fertilización en cada sitio, teniendo en cuenta observándose la diferencia y/o diversidad de topografía (clima, suelo y metros sobre nivel del mar).

En el experimento durante la estación lluviosa, fue el más mayor para el híbrido AV9139 con $50 \mathrm{kgN} / \mathrm{ha}$, se pudo establecer la producción con bajos insumos.

\section{CONCLUSIONES}

Se observó una alta fertilidad del suelo, teniendo en cuenta altos valores del $\mathrm{pH}\left(\mathrm{H}_{2} \mathrm{O}\right)$, $\mathrm{CE}, \mathrm{MO}, \mathrm{CIC}, \mathrm{Ca}$ y $\mathrm{Mg}$ intercambiable en la superficie.

Así mismo se observó un bajo valor para el $P$, de $0,50 \mathrm{mg} / \mathrm{kg}$ en la superficie.

De los resultados obtenidos de la calicata del suelo (desde la superficie a $50 \mathrm{~cm}$ de la profundidad), aumentó el valor del Ca y $\mathrm{Mg}$ intercambiable y el de la CIC de acuerdo con más profundidad, mientras que para el $\mathrm{K}$ la tendencia es inversa.

Especialmente, se observó alto valor para la CIC, y fue de 40 a $50 \mathrm{cmol} / \mathrm{kg}$ en todas las profundidades.

Como característica agronómica, para la altura de los tres híbridos, se observó el más alto para el AV 9139.

Al igual que el caso de la altura, para longitud de hoja, fue el más alto valor para el AV 9139.
Como característica agronómica, se observó la diferencia significativa al 1\% para no sólo para el híbrido sino también para la interacción (híbrido por nitrógeno), con respecto a la longitud de hoja el valor más alto fue para el AV 9139.

El rendimiento del grano con $14 \%$ de la humedad, se observó una diferencia significativa al 5\% para la interacción.

Aunque el rendimiento más alto fue para el AV9139, donde se observó una tendencia negativa con respecto a un mayor nivel de nitrógeno.

Se recomienda el AV 9139 con nivel de $50 \mathrm{kgN} / \mathrm{ha}$ además de $50 \mathrm{kgP}_{2} \mathrm{O}_{5} \quad \mathrm{y}$ $50 \mathrm{kgK}_{2} \mathrm{O} / \mathrm{ha}$, tomando en cuenta el más alto beneficio neto observado.

Se recomienda experimentar con otros híbridos para mantener la biodiversidad y prevenir riesgos de contaminación por plagas y enfermedades.

\section{REFERENCIAS}

1. Galeano Lobos ML. Evaluación del rendimiento del cultivo de maíz (Zea mays) variedad híbrida TNH 0032100 y TNH 0033100, con diferentes niveles de aplicación de Nitrógeno. pp 62. (Tesis de la Facultad de Ciencias Agropecuarias y Desarrollo Rural, Universidad Nacional de Pilar en el Departamento de Ñeembucú, Paraguay. 2012.

2. Valarezo Torres PV. Evaluación de tres híbridos de maíz Zea mays L. con tres distancias de siembra (Bachelor's thesis, Facultad de Ciencias Agrarias Universidad de Guayaquil). 2017.

3. Valery A, Reyes I. Evaluación de rizobacterias promotoras del crecimiento bajo diferentes esquemas de fertilización en el cultivo de maíz variedad HIMECA95. Revista colombiana de biotecnologia, 2013: 15(2), 81-8. 